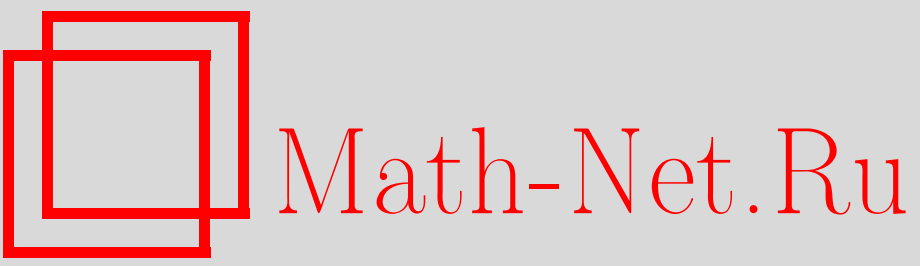

Ю. А. Кашлев, Локальная стохастическая теория каналирования. Кинетические функции в условиях взаимодействия быстрых частиц с атомами решетки, ТМФ, 2004, том 140, номер 1, 86-99

DOI: https://doi.org/10.4213/tmf78

Использование Общероссийского математического портала Math-Net.Ru подразумевает, что вы прочитали и согласны с пользовательским соглашением

http: //www . mathnet.ru/rus/agreement

Параметры загрузки:

IP : 3.85 .183 .62

26 апреля 2023 г., 18:32:35 
ТЕОРЕТИЧЕСКАЯ

И МАТЕМАТИЧЕСКАЯ

ФИЗИКА

Том 140, № 1

июль, 2004

(C) 2004 г.

\section{ЛОКАЛЬНАЯ СТОХАСТИЧЕСКАЯ ТЕОРИЯ КАНАЛИРОВАНИЯ. КИНЕТИЧЕСКИЕ ФУНКЦИИ В УСЛОВИЯХ ВЗАИМОДЕЙСТВИЯ БЫСТРЫХ ЧАСТИЦ С АТОМАМИ РЕШЕТКИ}

На основе аналитических методов теории марковских процессов, в том числе с использованием локального уравнения Фоккера-Планка, исследовано движение высокоэнергетических частиц в кристаллах с учетом их взаимодействия с тепловыми колебаниями решетки. Построена локальная матрица случайных воздействий, на базе которой дан вывод основных кинетических функций в пространстве поперечной энергии: $a\left(\varepsilon_{\perp}\right)$ - энергетических потерь за счет динамического трения и $b\left(\varepsilon_{\perp}\right)$ - дифффузионной функции. Показано, что особенности функций $a\left(\varepsilon_{\perp}\right)$ и $b\left(\varepsilon_{\perp}\right)$ связаны с различием вкладов в кинетику от частиц, движущихся в трех разных режимах: каналирования, квазиканалирования и хаотического движения.

Ключевые слова: стохастическая теория, марковский процесс, плоскостное каналирование, энергетические потери, поперечная энергия.

\section{1. ВВЕДЕНИЕ}

В задачах неравновесной статистической механики (броуновское движение частиц в жидкости, броуновское движение квантового осциллятора, релаксация в системе осцилляторов) рассматривается эволюция малой подсистемы в условиях случайного процесса. Для выделенной подсистемы в условиях слабого взаимодействия между подсистемами вводятся стохастические дифференциальные уравнения и соответствуюшее им уравнение Фоккера-Планка с последующим применением математического аппарата теории марковских процессов. Впервые такой подход был использован в работе [1], где принята традиционная постановка задачи Ланжевена с феноменологическим коэффициентом трения. В дальнейшем эти же уравнения использовались для описания броуновского движения тяжелой частицы в жидкости [2], где впервые было дано определение коэффициента трения через корреляционную функцию случайных сил. В более поздних исследованиях [3], [4] динамическое трение выражалось через запаздываюшую функцию Грина "сила-сила".

* Институт металлургии и материаловедения им. А. А. Байкова РАН, Москва, Россия 
В предлагаемой работе на примере быстрых частищ, взаимодействующих с тепловыми колебаниями атомов решетки, установлено, что не только энергетические потери частиц, но и диффузионная функция в пространстве поперечной энергии может быть представлена в форме интегрального преобразования локальной матрицы случайных воздействий. Указанное представление можно расценивать как развитие традиционного подхода, используемого в теории движения атомных частищ в среде.

Ограничимся анализом движения быстрых частиц между двумя кристаллографическими плоскостями (эффект плоскостного каналирования [5]). При выбранной таким образом геометрии основным приближением становится приближение непрерывного потенциала плоскости, которое состоит в следуюшем. Пусть положительно заряженные частицы влетают в кристалл под углом $\psi_{0}$ по отношению к кристаллографической плоскости, образуюшей стенку канала. В этом случае частицы, лежащие на отрезке длиной $\left(m v_{0} \psi_{0}\right)^{-1}$, когерентно взаимодействуют со всеми атомами плоскости (здесь принята система единиц, в которой $\hbar=k_{\mathrm{B}}=1 ; m$ и $v_{0}$ - масса и скорость быстрой частицы). Если указанная длина велика по сравнению с межатомным расстоянием, то $\mathrm{c}$ точки зрения рассеяния расположение атомов на ней несушественно. Поэтому в первом приближении достаточно учитывать суммарный потенциал плоскости, усредненный по расположению атомов. Будем считать, что ось $x$ перпендикулярна плоскости канала $(y, z)$, а ось $z$ направлена вдоль пучка частиц. Тогда непрерывный потенциал плоскости является функцией поперечной относительно стенки канала координаты $U_{\mathrm{pl}}(x)$. Явньй вид $U_{\mathrm{pl}}(x)$ представлен ниже.

\section{2. ЛОКАЛЬНАЯ МАТРИЦА СЛУЧАЙНЫХ ВОЗДЕЙСТВИЙ В СЛУЧАЕ УЧЕТА ПРОСТРАНСТВЕННЫХ КОРРЕЛЯЦИЙ}

Плотность частиц в данной точке изменяется с течением времени. Если рассматривать пространственно-временные корреляции, то значение плотности в точке $\mathbf{r}$ в момент времени $t$ оказывает влияние на величину плотности в точке $\mathbf{r}^{\prime}$ в момент $t+t^{\prime}$. Тем не менее в частных случаях учет двух видов корреляции не всегда оправдан. Например, при движении быстрых частиц в электронном газе доминируюшую роль играют корреляции во времени [6]. Это хорошо объяснимо, когда частицы с энергией порядка нескольких МэВ рассеиваются на электронах и скорости частищ близки к скорости Ферми. Однако в случае рассеяния таких частиц на тепловых колебаниях атомов решетки физическая ситуация совсем другая. В этом случае скорость тепловых движений атомов решетки на несколько порядков величины меньше скорости частиц, так что движением атомов в период прохождения частишы через кристалл можно пренебречь. Другими словами, можно рассматривать движение частиц в статической решетке с "замороженным" разбросом атомов и ограничиться учетом одного вида корреляций - пространственных. Далее рассматривается только этот случай.

В теории релаксационных процессов [6]-[8] воздействие решетки с "замороженным" разбросом атомов описывается либо с помощью локальной стохастической силы $\tilde{f}(\mathbf{r})$, либо с помошью ее классического аналога - флуктуационной составляющей силы $\delta f(\mathbf{r})=f(\mathbf{r})-\langle f(\mathbf{r})\rangle_{\mathrm{G}}$, где усреднение $\langle\ldots\rangle_{\mathrm{G}}$ выполняется по нормальному распределению. С учетом сделанных замечаний корреляшионную функцию “сила-сила” запишем в 
виде

$$
\begin{aligned}
\Psi_{\alpha \beta}\left(\mathbf{q}, v_{0} t ; \mathbf{q}^{\prime}, v_{0} t^{\prime}\right)= & \sum_{\nu, \mu}\left\{\left\langle f_{\alpha}\left(\mathbf{q}-\mathbf{q}_{\mathrm{c}}^{\nu}, v_{0} t-z_{\mathrm{c}}^{\nu}\right) f_{\beta}\left(\mathbf{q}^{\prime}-\mathbf{q}_{\mathrm{c}}^{\mu}, v_{0} t^{\prime}-z_{\mathrm{c}}^{\mu}\right)\right\rangle_{\mathrm{G}}-\right. \\
& \left.-\left\langle f_{\alpha}\left(\mathbf{q}-\mathbf{q}_{\mathrm{c}}^{\nu}, v_{0} t-z_{\mathrm{c}}^{\nu}\right)\right\rangle_{\mathrm{G}}\left\langle f_{\beta}\left(\mathbf{q}^{\prime}-\mathbf{q}_{\mathrm{c}}^{\mu}, v_{0} t^{\prime}-z_{\mathrm{c}}^{\mu}\right)\right\rangle_{\mathrm{G}}\right\} .
\end{aligned}
$$

Обозначим $\mathbf{r}=\left(\mathbf{q}, v_{0} t\right), \mathbf{q}=(x, y), v_{0} t$-продольная координата частицы; $\mathbf{r}_{\mathrm{c}}^{\nu}=\left(\mathbf{q}_{\mathrm{c}}^{\nu}, z_{\mathrm{c}}^{\nu}\right)$, $\mathbf{q}_{\mathrm{c}}^{\nu}$ - координата $\nu$-го атома в поперечной (относительно оси $z$ ) плоскости, $z_{\mathrm{c}}^{\nu}-$ продольная координата атома решетки; индексы $\alpha$ и $\beta$ принимают значения $x, y$ и $z$. Если соотношение (1) проинтегрировать по времени, получится локальная матрица случайных воздействий среды на частицу, с помощью которой устанавливается связь микроскопической аналитической формы, т.е. корреляционной функции (1), с макроскопическими кинетическими функциями. Выполнив интегрирование по времени, получаем

$$
L_{\alpha \beta}=\int_{0}^{t} d t^{\prime} \Psi_{\alpha \beta}\left(\mathbf{q}, v_{0} t ; \mathbf{q}^{\prime}, v_{0} t^{\prime}\right) .
$$

Выражение (2) с учетом (1) запишем в виде

$$
\begin{gathered}
L_{\alpha \beta}=L_{\alpha \beta}^{(1)}+L_{\alpha \beta}^{(2)}, \quad L_{\alpha \beta}^{(2)}=K_{\alpha \beta}-R_{\alpha \beta}, \\
L_{\alpha \beta}^{(1)}=\sum_{\nu}\left\langle f_{\alpha}\left(\mathbf{q}-\mathbf{q}_{\mathrm{c}}^{\nu}, v_{0} t-z_{\mathrm{c}}^{\nu}\right) \int_{0}^{t} d t^{\prime} f_{\beta}\left(\mathbf{q}^{\prime}-\mathbf{q}_{\mathrm{c}}^{\nu}, v_{0} t^{\prime}-z_{\mathrm{c}}^{\nu}\right)\right\rangle_{\mathrm{G}} \\
K_{\alpha \beta}=\sum_{\nu \mu}^{\prime}\left\langle f_{\alpha}\left(\mathbf{q}-\mathbf{q}_{\mathrm{c}}^{\nu}, v_{0} t-z_{\mathrm{c}}^{\nu}\right) \int_{0}^{t} d t^{\prime} f_{\beta}\left(\mathbf{q}^{\prime}-\mathbf{q}_{\mathrm{c}}^{\mu}, v_{0} t^{\prime}-z_{\mathrm{c}}^{\mu}\right)\right\rangle_{\mathrm{G}} \\
R_{\alpha \beta}=\sum_{\nu \mu}\left\langle f_{\alpha}\left(\mathbf{q}-\mathbf{q}_{\mathrm{c}}^{\nu}, v_{0} t-z_{\mathrm{c}}^{\nu}\right)\right\rangle_{\mathrm{G}} \int_{0}^{t} d t^{\prime}\left\langle f_{\beta}\left(\mathbf{q}^{\prime}-\mathbf{q}_{\mathrm{c}}^{\mu}, v_{0} t^{\prime}-z_{\mathrm{c}}^{\mu}\right)\right\rangle_{\mathrm{G}}
\end{gathered}
$$

Предполагая, что кристалл близок к состоянию статистического равновесия, введем равновесную плотность вероятности распределения атомов решетки в поперечной плоскости $w_{\perp}\left(\mathbf{q}_{\mathrm{c}}^{\nu}\right)$ и плотность в $z$-направлении $w_{z}\left(z_{\mathrm{c}}^{\nu}\right)$. Далее определим разность продольных координат $v_{0} \tau=v_{0}\left(t-t^{\prime}\right)$ и заменим $\mathbf{q}^{\prime}$ на $\mathbf{q}-\mathbf{v}_{\perp} \tau$, где $\mathbf{v}_{\perp}-$ поперечная скорость частицы. В результате матрица (3a) примет вид

$$
\begin{aligned}
L_{\alpha \beta}^{(1)}= & \int d \mathbf{q}_{\mathrm{c}} w_{\perp}\left(\mathbf{q}_{\mathrm{c}}\right) v_{0} \int_{-\infty}^{\infty} d t_{1} w_{z}\left(v_{0} t_{1}\right) f_{\alpha}\left(\mathbf{q}-\mathbf{q}_{\mathrm{c}}, v_{0}\left(t-t_{1}\right)\right) \times \\
& \times \int_{0}^{t} d \tau f_{\beta}\left(\mathbf{q}-\mathbf{q}_{\mathrm{c}}-\mathbf{v}_{\perp} \tau, v_{0}\left(t-t_{1}-\tau\right)\right),
\end{aligned}
$$

где $v_{0} t_{1}=z_{\mathrm{c}}^{\nu}$. Выполнив те же преобразования в (3б), получим

$$
\begin{aligned}
K_{\alpha \beta}= & \int d \mathbf{q}_{\mathrm{c}}^{(1)} \int d \mathbf{q}_{\mathrm{c}}^{(2)} \widetilde{P}_{\perp}\left(\mathbf{q}_{\mathrm{c}}^{(1)}, \mathbf{q}_{\mathrm{c}}^{(2)}\right) v_{0}^{2} \int_{-\infty}^{\infty} d t_{1} \int_{-\infty}^{\infty} d t_{2} \widetilde{P}_{z}\left(v_{0} t_{1}, v_{0} t_{2}\right) \times \\
& \times f_{\alpha}\left(\mathbf{q}-\mathbf{q}_{\mathrm{c}}^{(1)}, v_{0}\left(t-t_{1}\right)\right) \int_{0}^{t} d \tau f_{\beta}\left(\mathbf{q}-\mathbf{q}_{\mathrm{c}}^{(2)}-\mathbf{v}_{\perp} \tau, v_{0}\left(t-t_{2}-\tau\right)\right),
\end{aligned}
$$


где $\widetilde{P}_{z}\left(v_{0} t_{1}, v_{0} t_{2}\right)$ - двухчастичная функция нормального распределения атомов решетки по продольным координатам, $\widetilde{P}_{\perp}\left(\mathbf{q}_{\mathrm{c}}^{(1)}, \mathbf{q}_{\mathrm{c}}^{(2)}\right)$ - та же функция в поперечной плоскости. Матрица (3в) после тех же подстановок, что и при выводе формулы (4), будет отличаться от (5) только тем, что вместо $\widetilde{P}_{\perp}\left(\mathbf{q}_{\mathrm{c}}^{(1)}, \mathbf{q}_{\mathrm{c}}^{(2)}\right) \widetilde{P}_{z}\left(v_{0} t_{1}, v_{0} t_{2}\right)$ появится произведение одночастичных функций $\widetilde{P}_{\perp}\left(\mathbf{q}_{\mathrm{c}}^{(1)}\right) \widetilde{P}\left(\mathbf{q}_{\mathrm{c}}^{(2)}\right) w_{z}\left(v_{0} t_{1}\right) w_{z}\left(v_{0} t_{2}\right)$. Если потенциал взаимодействия быстрой частицы с атомом решетки обозначить $V(\mathbf{r})$, то с помощью формул (3)-(5) нетрудно показать, что

$$
\begin{gathered}
L_{\alpha \beta}(\mathbf{r} ; t)=L_{\alpha \beta}^{(1)}(\mathbf{r} ; t)+L_{\alpha \beta}^{(2)}(\mathbf{r} ; t), \\
L_{\alpha \beta}^{(1)}(\mathbf{r} ; t)=\int d \mathbf{r}_{\mathrm{c}} W_{1}\left(\mathbf{r}_{\mathrm{c}}\right) \nabla_{\alpha} V\left(\left|\mathbf{r}-\mathbf{r}_{\mathrm{c}}\right|\right) \int_{0}^{t} d \tau \nabla_{\beta} V\left(\left|\mathbf{r}-\mathbf{r}_{\mathrm{c}}-\mathbf{v}_{0} \tau\right|\right), \\
L_{\alpha \beta}^{(2)}(\mathbf{r} ; t)=\int d \mathbf{r}_{\mathrm{c}}^{(1)} \int d \mathbf{r}_{\mathrm{c}}^{(2)} g\left(\mathbf{r}_{\mathrm{c}}^{(1)}, \mathbf{r}_{\mathrm{c}}^{(2)}\right) \nabla_{\alpha} V\left(\left|\mathbf{r}-\mathbf{r}_{\mathrm{c}}^{(1)}\right|\right) \times \\
\times \int_{0}^{t} d \tau \nabla_{\beta} V\left(\left|\mathbf{r}-\mathbf{r}_{\mathrm{c}}^{(2)}-\mathbf{v}_{0} \tau\right|\right) .
\end{gathered}
$$

В формулах (6) использованы следуюшие обозначения:

$$
W_{1}\left(\mathbf{r}_{\mathrm{c}}\right)=w_{\perp}\left(\mathbf{q}_{\mathrm{c}}\right) w_{z}\left(v_{0} t_{1}\right)
$$

- одночастичная функция нормального распределения атомов,

$$
g\left(\mathbf{r}_{\mathrm{c}}^{(1)}, \mathbf{r}_{\mathrm{c}}^{(2)}\right)=W_{1}\left(\mathbf{q}_{\mathrm{c}}^{(1)}, v_{0} t_{1}\right) W_{1}\left(\mathbf{q}_{\mathrm{c}}^{(2)}, v_{0} t_{2}\right)-\widetilde{P}_{\perp}\left(\mathbf{q}_{\mathrm{c}}^{(1)}, \mathbf{q}_{\mathrm{c}}^{(2)}\right) \widetilde{P}_{z}\left(v_{0} t_{1}, v_{0} t_{2}\right)
$$

- парная корреляционная функция, $\nabla_{\alpha}=\partial / \partial r_{\alpha}$.

\section{3. ДИАГОНАЛЬНЫЙ ЭЛЕМЕНТ ЛОКАЛЬНОЙ МАТРИЦЫ ВОЗДЕЙСТВИЙ В УСЛОВИЯХ МАРКОВСКОГО ПРОЦЕССА}

Строго говоря, математический аппарат теории марковских процессов применим к системам, для которых внешнее воздействие представляет собой дельта-коррелированный случайный процесс [9]. Однако область применения этой теории значительно шире. В частности, она может использоваться для анализа случайного процесса с нулевым средним значением, когда корреляционная функция сил является экспоненциальной. Конечное время корреляции случайных воздействий не исключает возможности применения формализма марковских процессов, но только при определенных условиях. Чтобы пояснить эти условия, введем два времени релаксации: $\tau_{\mathrm{k}}$ - корреляционное время сил, которое по порядку величины равно длительности одного соударения, и $\tau_{\mathrm{c}}-$ корреляционное время скоростей частиц, которое по порядку величины можно оценить как время свободного пробега. Случайный процесс воздействия среды на частицу можно считать марковским для $\delta t \gg \Delta$, если при всех значениях $\left(x, \varepsilon_{\perp}\right)$ величина $\Delta$ удовлетворяет неравенствам

$$
\min \tau_{\mathrm{c}}>\Delta \gg \tau_{\mathrm{k}} .
$$


Левое неравенство из $(7)$ означает, что импульс частицы за время $\Delta$ изменяется незначительно. Правое неравенство означает, что прирашения импульса на неперекрывающихся интервалах времени длительности $\Delta$ являются независимыми. Из теории твердых тел [10] известно, что время релаксации $\tau_{\mathrm{k}}$ является наименьшим, так что условие $\tau_{\mathrm{c}} \gg \tau_{\mathrm{k}}$ хорошо выполняется. А это дает возможность найти такой интервал $\Delta$, что будут выполнены оба условия (7). Только в том случае, когда выполнены неравенства (7), оправдано само использование кинетических уравнений и, в частности, уравнения Фоккера-Планка, являюшегося составной частью математического аппарата теории марковских процессов.

Введем дополнительную аппроксимацию. Согласно (7) временно́й интервал $\delta t$ определяет верхний предел интегрирования по времени. Поскольку этот интервал является наибольшим, его можно положить равным бесконечности. На этом основании во всех последующих формулах матрица (6) заменена на ее предельное значение - стационарную матрищу

$$
L_{\alpha \beta}(\mathbf{r})=\lim _{t \rightarrow \infty} L_{\alpha \beta}(\mathbf{r} ; t)
$$

Теперь преобразуем выражения (6). Будем считать, что распределение атомов решетки в плоскости канала является однородным. Тогда можно считать, что элементарные акты рассеяния частиц обусловлены только поперечными смешениями атомов решетки $u_{x}$ и в матрице (6) основным является ее диагональный элемент $\alpha=\beta=x$. Учитывая, что $W_{1}\left(\mathbf{r}_{\mathrm{c}}\right)=n_{\mathrm{c}} 2 l P_{\perp}\left(x_{\mathrm{c}}\right)$, где $P_{\perp}\left(x_{\mathrm{c}}\right)$ - одночастичное распределение атомов по поперечной координате и $n_{\mathrm{c}}$ - плотность атомов решетки, диагональный элемент матрицы воздействий запишем в виде

$$
L_{x x}^{(1)}(\mathbf{r})=n_{\mathrm{c}} 2 l \int d \mathbf{r}_{\mathrm{c}} P_{\perp}\left(x_{\mathrm{c}}\right) \nabla_{x} V\left(\left|\mathbf{r}-\mathbf{r}_{\mathrm{c}}\right|\right) \int d \tau \nabla_{x} V\left(\left|\mathbf{r}-\mathbf{r}_{\mathrm{c}}-\mathbf{v}_{0} \tau\right|\right) .
$$

В случае кристаллической структуры с постоянным межплоскостным расстоянием $2 l$ одночастичное распределение равно $P_{\perp}\left(x_{\mathrm{c}}\right)=\sum_{n} \varphi\left(x_{\mathrm{c}}-n 2 l\right)$, и поскольку усреднение в (1) $-(3)$ выполняется по гауссовскому ансамблю, имеем

$$
\varphi(x)=\left(2 \pi \sigma_{\theta}^{2}\right)^{-1 / 2} \exp \left\{-\frac{x^{2}}{2 \sigma_{\theta}^{2}}\right\}
$$

где $\sigma_{\theta}$ - среднеквадратичное отклонение атома в направлении, перпендикулярном плоскости канала. Наконец, периодическую функцию $P_{\perp}\left(x_{\mathrm{c}}\right)$ представим в виде ряда Фурье

$$
2 l P_{\perp}\left(x_{\mathrm{c}}\right)=\sum_{n} G_{n} e^{i p_{n} x_{\mathrm{c}}}
$$

Здесь $p_{n}=\pi n / l, n$ - целые числа, $G_{n}=\exp \left\{-\left(p_{n}^{2} \sigma_{\theta}^{2} / 2\right)\right\}$.

В качестве потенциала взаимодействия быстрой частищы с атомом выберем мольеровскую аппроксимацию потенциала Томаса-Ферми

$$
V_{\mathrm{M}}(r)=V_{\mathrm{C}}(r) \sum_{i} \alpha_{i} e^{-\varkappa_{i} r}, \quad V_{\mathrm{C}}(r)=\frac{Z_{1} Z_{2} e^{2}}{r}
$$


где $\alpha_{1}=0.1 ; \alpha_{2}=0.55 ; \alpha_{3}=0.35 ; \beta_{1}=6.0 ; \beta_{2}=1.2 ; \beta_{3}=0.3 ; \varkappa_{i}=\beta_{i} / a_{\mathrm{TF}} ; a_{\mathrm{TF}}-$ радиус экранирования Томаса-Ферми, $Z_{1}$ и $Z_{2}$ - атомные номера частицы и атома решетки, соответственно; $i=1,2,3$. Принимая во внимание явный вид потенциала $V_{\mathrm{M}}(r)$ и распределения (9), а также учитывая взаимодействие частицы с ближайшей $x$-цепочкой атомов решетки, матричный элемент (8) преобразуем к виду

$$
L_{x x}^{(1)} \equiv L^{(1)}(x)=\frac{1}{v_{0}} 8 \pi\left(Z_{1} Z_{2} e^{2}\right)^{2} n_{\mathrm{c}} \sum_{n} I_{n}^{(1)} G_{n} \cos \left(p_{n} x\right),
$$

где

$$
\begin{gathered}
I_{n}^{(1)}=\sum_{i j} \alpha_{i} \alpha_{j} \int_{0}^{\Lambda_{1}} d k k^{3}\left(k^{2}+\varkappa_{i}^{2}\right)^{-1} \gamma_{n j}(k)\left\{\tilde{\beta}_{n j}(k)\left[\tilde{\beta}_{n j}(k)+\gamma_{n j}(k)\right]\right\}^{-1}, \\
\gamma_{n j}(k)=2\left(k^{2}+p_{n}^{2}+\varkappa_{j}^{2}\right), \quad \tilde{\beta}_{n j}(k)=\left|\left(\gamma_{n j}(k)\right)^{2}-\left(4 p_{n} k\right)^{2}\right|^{1 / 2},
\end{gathered}
$$

$\Lambda_{1}=\left[2\left(Z_{1} Z_{2} e^{2}\right)(M+m)\right]^{-1} M m v_{0}^{2}, M-$ масса атома решетки.

В квазигармоническом приближении парная корреляционная функция, вошедшая в (6), имеет вид

$$
g\left(r_{\mathrm{c}}^{(1)}, r_{\mathrm{c}}^{(2)}\right)=n_{\mathrm{c}} 2 l \tilde{g}\left(x_{\mathrm{c}}^{(1)}, x_{\mathrm{c}}^{(2)}\right)=-n_{\mathrm{c}} 2 l \sum_{n} \varphi\left(x_{\mathrm{c}}^{(1)}-n 2 l\right) \varphi\left(x_{\mathrm{c}}^{(2)}-n 2 l\right) .
$$

С учетом этой аппроксимации получим

$$
\begin{aligned}
L_{x x}^{(2)}(\mathbf{r})= & -2 n_{\mathrm{c}} 2 l \sum_{n} \int d \mathbf{r}_{\mathrm{c}}^{(1)} \int d \mathbf{r}_{\mathrm{c}}^{(2)} \varphi\left(x_{\mathrm{c}}^{(1)}-n 2 l\right) \varphi\left(x_{\mathrm{c}}^{(2)}-n 2 l\right) \times \\
& \times \Phi\left(\left|\boldsymbol{\lambda}_{\mathrm{c}}^{(1)}-\boldsymbol{\lambda}_{\mathrm{c}}^{(2)}\right|, 2 \sigma_{\theta}^{2}\right) \nabla_{x} V_{\mathrm{M}}\left(\left|\mathbf{r}-\mathbf{r}_{\mathrm{c}}^{(1)}\right|\right) \int_{0}^{\infty} d \tau \nabla_{x} V_{\mathrm{M}}\left(\left|\mathbf{r}-\mathbf{r}_{\mathrm{c}}^{(2)}-\mathbf{v}_{0} \tau\right|\right),
\end{aligned}
$$

где

$$
\mathbf{r}_{\mathrm{c}}=\left(x_{\mathrm{c}}, \boldsymbol{\lambda}_{\mathrm{c}}\right), \quad \Phi\left(\left|\boldsymbol{\lambda}_{\mathrm{c}}^{(1)}-\boldsymbol{\lambda}_{\mathrm{c}}^{(2)}\right|, 2 \sigma_{\theta}^{2}\right)=\left(4 \pi \sigma_{\theta}^{2}\right)^{-1} \exp \left(-\frac{\left|\boldsymbol{\lambda}_{\mathrm{c}}^{(1)}-\boldsymbol{\lambda}_{\mathrm{c}}^{(2)}\right|^{2}}{4 \sigma_{\theta}^{2}}\right) .
$$

Подставляя выражения $\varphi(x)$ и $V_{\mathrm{M}}(r)$ в (11), получаем

$$
L_{x x}^{(2)} \equiv L^{(2)}(x)=-\frac{1}{v_{0}} 8 \pi\left(Z_{1} Z_{2} e^{2}\right)^{2} n_{\mathrm{c}} \sum_{n} I_{n}^{(2)}(x),
$$

где

$$
\begin{aligned}
I_{n}^{(2)}(x)= & \frac{l}{\pi} \sigma_{\theta}^{-2} \int_{0}^{\Lambda_{1}} d k \exp \left(-k^{2} \sigma_{\theta}^{2}\right)\left\{\sum_{i} \alpha_{i} \int_{-\infty}^{\infty} d x^{\prime}\left(\gamma_{0 i}(k)\right)^{-1 / 2} \times\right. \\
& \left.\times \exp \left[-x^{\prime}\left(\gamma_{0 i}(k)\right)^{1 / 2}-\frac{\left(x+x^{\prime}-n 2 l\right)^{2}}{2 \sigma_{\theta}^{2}}\right]\right\}^{2} .
\end{aligned}
$$


Как основной член матрищы воздействий (10), так и коррелящионная поправка (12) имеют сложный аналитический вид. Поэтому для наглядности приведем графическое изображение безразмерного матричного элемента $\eta(x)=L(x) /(\mathrm{Jm})$, в котором

$$
J=8 \pi\left(Z_{1} Z_{2} e^{2}\right)^{2} n_{\mathrm{c}}\left(m v_{0}\right)^{-1} \text {. }
$$

Графики функции $\eta(\tilde{x})$, где $\tilde{x}=x / l$, представлены на рис. 1 при трех температурах $T=$ $300,500,700 \mathrm{~K}^{1}$. Функция $\eta(\tilde{x})$ симметрична, и на рисунке изображена только ее правая часть. Введенный здесь матричный элемент с достаточной степенью точности может быть аппроксимирован с помошью гауссовских функций

$$
\eta(x)=\left(\frac{300 K}{2 \pi T}\right)^{1 / 2}\left\{\exp \left(-\frac{\xi^{2} x^{2}}{2}\right)-\frac{1}{5 \xi x_{0}} \exp \left(-\frac{\xi^{2}\left(x-x_{0}\right)^{2}}{2}\right)\right\}
$$

если считать, что $1 / \xi=l$ и $\xi x_{0}=\sqrt{2}$. В общем случае величину $1 / \xi$ следует интерпретировать как длину корреляции в $x$-направлении [8], [11]. Наш анализ функции $\eta(\tilde{x})$ свидетельствует о том, что в частном случае плоскостного канала роль параметра корреляции переходит к его полуширине $l$, определяющей максимальное отклонение частицы от центра канала в $x$-направлении.

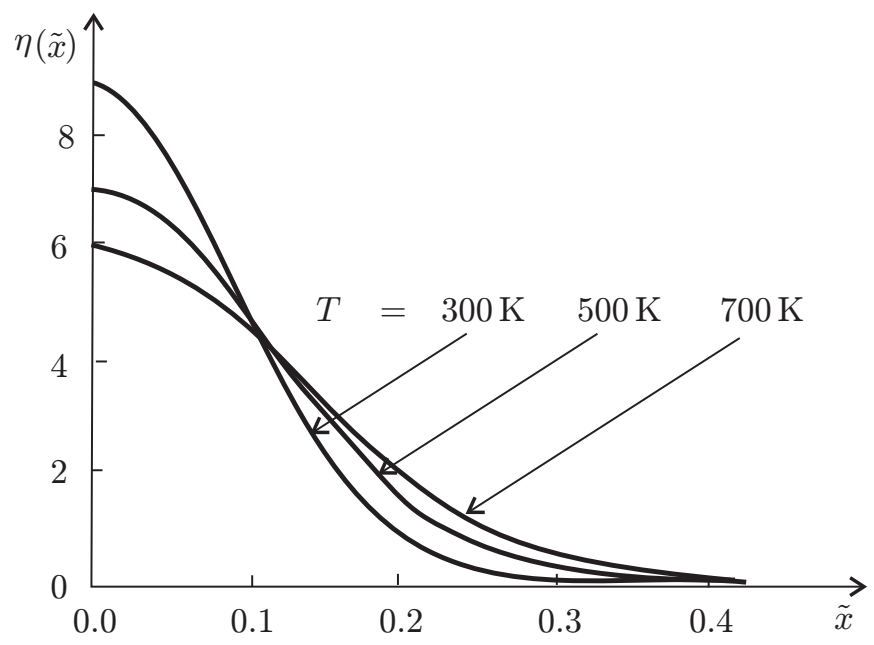

Рис. 1

Сделаем два замечания. Во-первых, теория корреляции в пространстве еще далека от завершения и в литературе, как правило, ограничиваются оценкой корреляционной длины. В нашем случае $1 / \xi \simeq 1 \AA$. Во-вторых, временное изменение матрицы случайных воздействий будет влиять на рассеяние частиц только в том случае, если время $\tau_{\xi}$, в

\footnotetext{
1) Расчет выполнен для $\alpha$-частиц с энергией $1.5 \mathrm{MэB}$, движущихся в плоскостном канале $(100)$ германия. Ширина канала $1.96 \AA$ А.
} 
течение которого рассеянная частица находится на длине корреляции, сравнимо с временем релаксации $\tau_{\mathrm{k}}(7)$. Однако оценка для $\alpha$-частиц показала, что в условиях рассеяния при комнатных температурах отношение $\tau_{\xi} / \tau_{\mathrm{k}}$ пренебрежимо мало, а следовательно, пренебрежимо мал вклад временнь́х корреляций. На наш взгляд, представленные здесь соображения подтверждают обоснованность приближения статических флуктуаций и статических корреляционных функций, которое используется в настоящей работе.

\section{4. КИНЕТИЧЕСКИЕ ФУНКЦИИ И КИНЕТИЧЕСКИЕ КОЭФФИЦИЕНТЫ}

Сформулируем основные уравнения кинетической теории каналирования. В условиях иерархии времен (7) движение быстрых частиц в кристалле является марковским процессом. Если в качестве переменной использовать поперечную энергию быстрой частицы

$$
\varepsilon_{\perp}=\frac{1}{2} m(\dot{x})^{2}+U_{\mathrm{pl}}(x),
$$

то уравнение для совместной плотности вероятности $\mathcal{P}\left(x, \varepsilon_{\perp}, t\right)$ может быть построено на базе системы стохастических уравнений, соответствуюших двумерному марковскому процессу $\left(x, \varepsilon_{\perp}\right)$,

$$
\begin{gathered}
\dot{x}=\left[\frac{2}{m}\left(\varepsilon_{\perp}-U_{\mathrm{pl}}(x)\right)\right]^{1 / 2}, \\
\dot{\varepsilon}_{\perp} \frac{1}{\left[(2 / m)\left(\varepsilon_{\perp}-U_{\mathrm{pl}}(x)\right)\right]^{1 / 2}}=-H\left(x \sqrt{\frac{2}{m}\left(\varepsilon_{\perp}-U_{\mathrm{pl}}(x)\right)}\right)+\tilde{f}(x) .
\end{gathered}
$$

Уравнение (13б) есть не что иное, как уравнение Ито ${ }^{2)}$. В него вошли дрейфовый член

$$
H\left(x, \sqrt{\frac{2}{m}\left(\varepsilon_{\perp}-U_{\mathrm{pl}}(x)\right)}\right),
$$

который соответствует систематической части силы, связанной с внешними полями, и $\tilde{f}(x)$ - случайная сила, обусловленная хаотическим движением атомов решетки.

Системе уравнений (13) соответствует статистически эквивалентное уравнение движения для совместной плотности вероятности $\mathcal{P}\left(x, \varepsilon_{\perp}, t\right)$, а именно уравнение Фоккера-Планка. Процедура перехода от (13) к уравнению Фоккера-Планка хорошо известна [8]. Поэтому сразу представим окончательно уравнение

$$
\begin{aligned}
\frac{\partial}{\partial t} \mathcal{P}\left(x, \varepsilon_{\perp}, t\right) & =L(x) \frac{\partial^{2}}{\partial \varepsilon_{\perp}^{2}}\left\{\frac{1}{m}\left(\varepsilon_{\perp}-U_{\mathrm{pl}}(x)\right) \mathcal{P}\left(x, \varepsilon_{\perp}, t\right)\right\}- \\
- & \frac{\partial}{\partial x}\left\{\left[\frac{2}{m}\left(\varepsilon_{\perp}-U_{\mathrm{pl}}(x)\right)\right]^{1 / 2} \mathcal{P}\left(x, \varepsilon_{\perp}, t\right)\right\}+ \\
+ & \frac{\partial}{\partial \varepsilon_{\perp}}\left\{\left[\frac{2}{m}\left(\varepsilon_{\perp}-U_{\mathrm{pl}}(x)\right)\right]^{1 / 2} H\left(x, \sqrt{\frac{2}{m}\left(\varepsilon_{\perp}-U_{\mathrm{pl}}(x)\right)}\right) \mathcal{P}\left(x, \varepsilon_{\perp}, t\right)\right\}- \\
- & \frac{1}{2} \frac{\partial}{\partial \varepsilon_{\perp}}\left\{\frac{1}{m} L(x) \mathcal{P}\left(x, \varepsilon_{\perp}, t\right)\right\} .
\end{aligned}
$$

2) Уравнение Ито записано не в традиционном, а в более удобном для нас виде (см. раздел 4.8 в [8]). Оно также известно как нелинейное уравнение Ланжевена. 
При переходе от (13) к (14) случайная сила $\tilde{f}(x)$, вызывающая фллуктуации поперечной энергии, была заменена ее классическим аналогом $\delta f(x)$. Локальная флуукуация силы $\delta f(x)=f(x)-\langle f(x)\rangle_{\mathrm{G}}$, представляюшая собой случайное отклонение от среднего значения, в статистической механике [12] рассматривается как случайная величина. Как правило, источником информации относительно флуктуации $\delta f(x)$ может быть ее момент - среднее значение произведения случайных величин или корреляшионная функция (1), описывающая корреляцию в точках $x$ и $x^{\prime}$. Диагональный элемент матрищы случайных воздействий $L(x)=L^{(1)}(x)+L^{(2)}(x)(10),(12)$, полученный на основе $(1),(2)$ с учетом геометрии плоскостного каналирования, вносит в уравнение (14) специфику движения быстрых частиц между кристаллографическими плоскостями.

Отметим, что такое же уравнение Фоккера-Планка, включаюшее матричные элементы (10), (12), может быть получено на основе обобщенного уравнения Больцмана в условиях плоскостного каналирования [13]. Для этого достаточно разложить подынтегральную функцию в члене столкновений с учетом того, что в результате столкновений частицы незначительно отклоняются от первоначального направления и движутся под мальми углами к кристаллографическим плоскостям.

Поскольку время протекания процесса в окрестности заданного значения $x$ обратно пропорционально скорости, условное распределение частиц при фиксированной поперечной энергии запишем в виде

$$
P_{\mathrm{c}}\left(x \mid \varepsilon_{\perp}\right)=\frac{1}{2 \zeta\left(\varepsilon_{\perp}\right)}\left[\frac{1}{m}\left(\varepsilon_{\perp}-U_{\mathrm{pl}}(x)\right)\right]^{-1 / 2},
$$

где

$$
U_{\mathrm{pl}}(x)<\varepsilon_{\perp} \quad \text { и } \quad \zeta\left(\varepsilon_{\perp}\right)=\frac{1}{2} \int d x\left[\frac{1}{m}\left(\varepsilon_{\perp}-U_{\mathrm{pl}}(x)\right)\right]^{-1 / 2} .
$$

Принимая во внимание, что вплоть до настоящего времени эффект каналирования изучался только в отсутствие внешних полей, отбросим дрейфовый член в уравнении Фоккера-Планка (14). Тогда, учитывая, что $\mathcal{P}\left(x, \varepsilon_{\perp}, t\right)=P\left(\varepsilon_{\perp}, t\right) P_{\mathrm{c}}\left(x \mid \varepsilon_{\perp}\right)$, а также формулу (15), представим искомое уравнение в виде

$$
\frac{1}{J} \frac{\partial}{\partial t} P\left(\varepsilon_{\perp}, t\right)=-\frac{\partial}{\partial \varepsilon_{\perp}}\left\{a\left(\varepsilon_{\perp}\right) P\left(\varepsilon_{\perp}, t\right)\right\}+\frac{\partial^{2}}{\partial \varepsilon_{\perp}^{2}}\left\{b\left(\varepsilon_{\perp}\right) P\left(\varepsilon_{\perp}, t\right)\right\} \varepsilon_{\perp}^{\mathrm{cr}},
$$

где $a\left(\varepsilon_{\perp}\right)$ и $b\left(\varepsilon_{\perp}\right)$ - безразмерные энергетические потери за счет динамического трения и безразмерная диффузионная функция, описывающая диффузию частиц в пространстве поперечной энергии. С учетом явного вида диагонального элемента матрицы случайных воздействий (10), (12) кинетические функции после ряда преобразований удается представить в форме

$$
\begin{aligned}
& a\left(\varepsilon_{\perp}\right)=\frac{m^{1 / 2}}{2 \zeta\left(\varepsilon_{\perp}\right)} \sum_{n} \int d x\left[\varepsilon_{\perp}-U_{\mathrm{pl}}(x)\right]^{-1 / 2}\left[I_{n}^{(1)} G_{n} \cos \left(p_{n} x\right)-I_{n}^{(2)}(x)\right], \\
& b\left(\varepsilon_{\perp}\right)=\frac{m^{1 / 2}}{\zeta\left(\varepsilon_{\perp}\right) \varepsilon_{\perp}^{\mathrm{cr}}} \sum_{n} \int d x\left[\varepsilon_{\perp}-U_{\mathrm{pl}}(x)\right]^{1 / 2}\left[I_{n}^{(1)} G_{n} \cos \left(p_{n} x\right)-I_{n}^{(2)}(x)\right] .
\end{aligned}
$$


Здесь $\varepsilon_{\perp}^{\mathrm{cr}}=E_{0} \psi_{\mathrm{cr}}^{2}-$ критическая поперечная энергия, $E_{0}$ - энергия быстрой частишы. Усредняя мольеровскую аппроксимацию $V_{\mathrm{M}}$ с одночастичной функцией распределения $P_{\perp}(x)$, запишем плоскостной потенциал в виде

$$
\begin{aligned}
U_{\mathrm{pl}}(x) & =n_{\mathrm{c}} 2 l \sum_{n} \int d x_{\mathrm{c}} \varphi\left(x_{\mathrm{c}}-n 2 l\right) \int d \lambda_{\mathrm{c}} V_{\mathrm{M}}\left(\sqrt{\lambda_{\mathrm{c}}^{2}+(x-n 2 l)^{2}}\right)= \\
& =4 \pi Z_{1} Z_{2} e^{2} 2 l a_{\mathrm{TF}}^{2} \sum_{i, n} G_{n}\left[\beta_{i}^{2}+p_{n}^{2} a_{\mathrm{TF}}^{2}\right]^{-1} \cos \left(p_{n} x\right) .
\end{aligned}
$$

Кинетические функции (16) определены в широком интервале поперечных энергий и имеют достаточно сложный вид. Графики функций $a\left(\varepsilon_{\perp}\right)$ и $b\left(\varepsilon_{\perp}\right)$ для $\alpha$-частиц в той же физической ситуации, что использована при построении кривых $\eta(x)$ (рис. 1), представлены на рис. $2: a\left(\varepsilon_{\perp}\right)$ - при трех температурах $300,500,700 \mathrm{~K}$ и $b\left(\varepsilon_{\perp}\right)-$ при $300 \mathrm{~K}$.

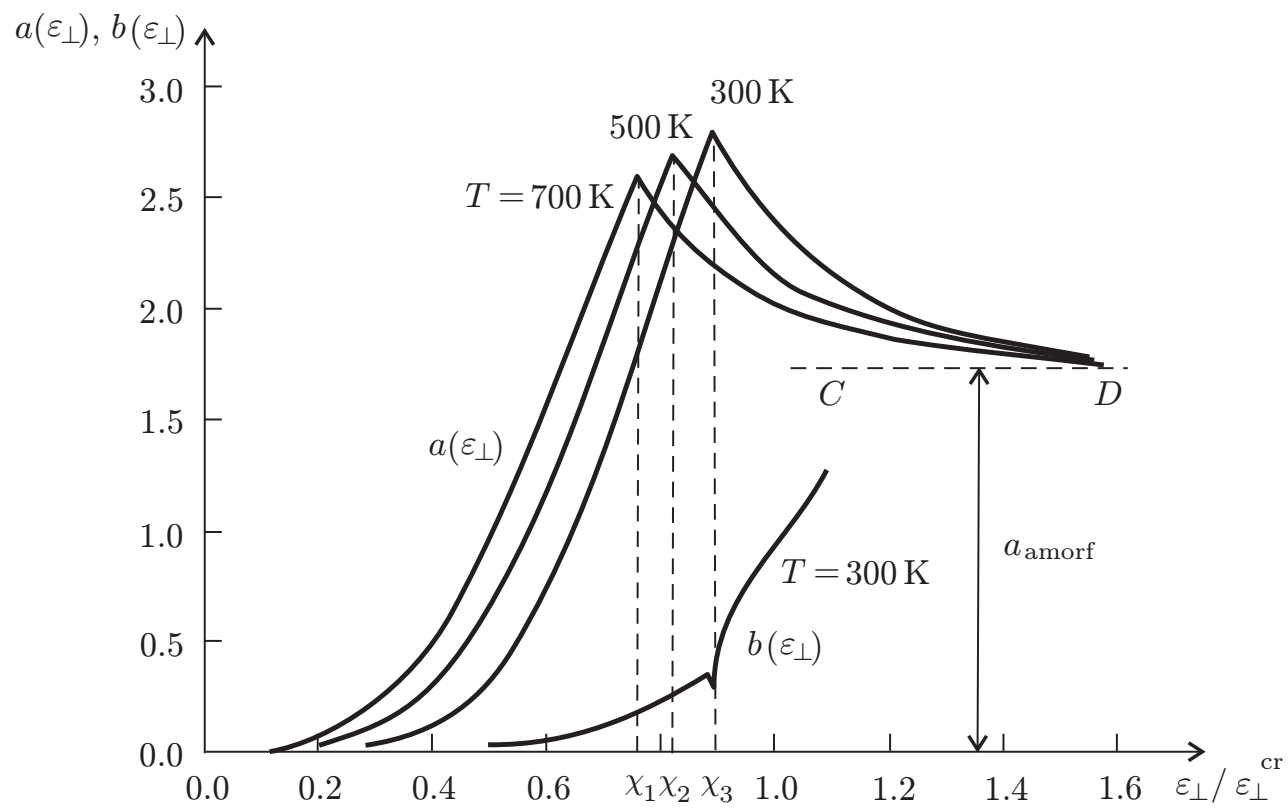

Рис. 2

Переход от кинетических функций к кинетическому коэффициенту выполним на примере одного из них - энергетических потерь хаотических частищ $A \equiv(-d E / d t)_{\text {random }}$. Будем рассматривать поперечные энергии частиш, характерные для хаотического пучка $2 \varepsilon_{\perp}^{\mathrm{cr}}>\varepsilon_{\perp}>\varepsilon_{\perp}^{\mathrm{cr}}$, и температуры $T \gtrsim \theta_{\mathrm{D}}$, где $\theta_{\mathrm{D}}-$ температура Дебая. Как видно из рис. 2 , функция $a\left(\varepsilon_{\perp}\right)$ спадает с ростом поперечной энергии как $\left(\varepsilon_{\perp}^{\mathrm{cr}} / \varepsilon_{\perp}\right)^{\nu}$, где $\nu<1$. Аналогично она уменьшается с ростом приведенной температуры. Таким образом, можно записать

$$
a\left(\varepsilon_{\perp}\right)=C_{2}\left(\frac{\varepsilon_{\perp}^{\mathrm{cr}}}{\varepsilon_{\perp}}\right)^{\nu}\left(\frac{T}{\theta_{\mathrm{D}}}\right)^{-\chi},
$$


где показатели степени $\nu$ и $\chi$, а также множитель $C_{2}$ определяются на основе графического изображения функции (для хаотических $\alpha$-частиц согласно рис. 2 имеем $\nu=3 / 4$, $\chi=1 / 3)$. Искомый кинетический коэффициент находим путем усреднения (18) в пространстве поперечной энергии. Переходя к размерной форме с помошью коэффищиента J, имеем

$$
A=C_{3}\left|V_{\mathrm{c}}(\bar{r})\right|^{2} I\left(\varepsilon_{\perp}^{\mathrm{cr}}\right) n_{\mathrm{c}}^{1 / 3} \frac{1}{v_{0} m}\left(\frac{T}{\theta_{\mathrm{D}}}\right)^{-\chi}
$$

где

$$
I\left(\varepsilon_{\perp}^{\mathrm{cr}}\right)=\left(\varepsilon_{\perp}^{\mathrm{cr}}\right)^{\nu} \int_{\varepsilon_{\perp}^{\mathrm{cr}}}^{\infty} d \varepsilon_{\perp} G\left(\varepsilon_{\perp}\right) \varepsilon_{\perp}^{-\nu},
$$

$G\left(\varepsilon_{\perp}\right)$ - плотность числа состояний частиц с поперечной энергией в интервале $\left(\varepsilon_{\perp}, \varepsilon_{\perp}+\right.$ $\left.d \varepsilon_{\perp}\right), \quad \bar{r}$ - среднее расстояние между атомами решетки, $(3 / 5) \bar{r}^{3}=n_{\mathrm{c}}^{-1}, C_{3}=40 C_{2}$. Конечно, полное квазиравновесное распределение, кроме плотности числа состояний, включает распределение $n\left(\varepsilon_{\perp}\right)=\Lambda e^{-F_{21} \varepsilon_{\perp}}$, где $\Lambda$ - абсолютная активность и $1 / F_{21}-$ поперечная квазитемпература частищ [14]. Однако для простоты математической записи в $(19)$ мы полагали $1 / F_{21} \rightarrow \infty$, что соответствует переходу от формул неравновесной статистической термодинамики к соотношениям теории Линдхарда [5].

Множитель $\left|V_{\mathrm{c}}(\bar{r})\right|^{2} I\left(\varepsilon_{\perp}^{\mathrm{cr}}\right)$ в (19) определяется видом потенциала взаимодействия. При этом эффект экранирования определяет величину $I\left(\varepsilon_{\perp}^{\mathrm{cr}}\right)$, поскольку критическая энергия $\varepsilon_{\perp}^{\mathrm{cr}}[5]$ связана с плоскостным потенциалом (17). Что касается температурной зависимости (19), то при $\chi<1$ она является достаточно слабой, и это не следует связывать с особенностью статической модели решетки, использованной при выводе формул $(10),(12)$ и (16). Для этого достаточно сравнить температурный ход (19) с зависимостью, которую дает динамическая модель решетки.

Вычислим потери хаотических частищ с учетом тепловых колебаний атомов решетки. Вероятность перехода в единицу времени для частиц хаотического пучка

$$
\widetilde{\mathcal{P}}(\mathbf{q}, \omega) d \mathbf{q} d \omega=-\frac{1}{(2 \pi)^{2}}|\widetilde{V}(q)|^{2} S(\mathbf{q}, \omega) \delta\left(\left(\mathbf{q} v_{0}\right)-\omega\right) d \mathbf{q} d \omega
$$

где

$$
\widetilde{V}(q)=\varkappa\left(\frac{\omega_{q}}{2 n_{\mathrm{c}} \bar{\Omega} M s^{2}}\right)^{1 / 2}
$$

- фурье-компонента потенциала взаимодействия частищы с колебаниями решетки, $\varkappa$ константа связи, $\bar{\Omega}$ - объем, $\omega_{q}$ - энергия фонона, $s$ - скорость звука,

$$
S(\mathbf{q}, \omega)=(2 \pi)^{-1} \int_{0}^{\infty} d t e^{i \omega t}\left\langle\pi_{-\mathbf{q}} \pi_{\mathbf{q}}(t)\right\rangle_{\text {eq }}
$$

- динамический формфактор,

$$
\left\langle\pi_{-\mathbf{q}} \pi_{\mathbf{q}}(t)\right\rangle_{\mathrm{eq}}=e^{-2 W} \sum_{\mathbf{R}} e^{-i \mathbf{q} \mathbf{R}}\langle(\mathbf{q u}(0,0)(\mathbf{q u}(\mathbf{R}, t)))\rangle_{\mathrm{eq}}
$$

Кроме того, использованы следующие обозначения: $\pi_{\mathbf{q}}-$ фурье-компонента фолуктуаций плотности, обусловленных тепловыми колебаниями, $e^{-2 W}-$ фактор Дебая-Валлера, 
$\langle\ldots\rangle_{\text {eq }}$ - усреднение по равновесному состоянию кристалла, $\mathbf{u}(\mathbf{R}, t)$ - смешение атома из узла $\mathbf{R}$ в момент времени $t$. Потери хаотических частиц за счет динамического трения можно представить в виде

$$
\tilde{A}=(2 \pi)^{3} \int_{0}^{\infty} d \omega \omega \int d \mathbf{q} \widetilde{\mathcal{P}}(\mathbf{q}, \omega) .
$$

На основе (20) и (21) после интегрирования по угловым переменным вектора q получаем

$$
\tilde{A}=2(2 \pi)^{-2} \bar{\Omega} v_{0} \int_{0}^{\infty} d \omega \int d q q^{3}|\widetilde{V}(q)|^{2} S(q, \omega)\left|\int_{0}^{\infty} d t^{\prime} e^{-i \omega t^{\prime}} J_{1}\left(q v_{0} t^{\prime}\right)\right|
$$

где $J_{1}(x)$ - цилиндрическая функция Бесселя. Как и при выводе формулы (19), будем рассматривать случай высоких скоростей, когда $E_{0} \gtrsim 1 \mathrm{MэВ.} \mathrm{Тогда}$

$$
\left|\int_{0}^{\infty} d t^{\prime} e^{-i \omega t^{\prime}} J_{1}\left(q v_{0} t^{\prime}\right)\right|=\pi \omega\left(q v_{0}\right)^{-2} .
$$

Корреляционную функцию “плотность-плотность", вошедшую в (20б), вычислим в нулевом приближении по потенциалу рассеяния. В этом приближении формфактор (20б) принимает простой вид

$$
S_{0}(q, \omega)=e^{-2 W} \frac{q^{2}}{2 m \omega_{q}}\left\{\tilde{\eta}\left(\omega_{q}\right) \delta\left(\omega-\omega_{q}\right)+\left(\tilde{\eta}\left(\omega_{q}\right)+1\right) \delta\left(\omega+\omega_{q}\right)\right\},
$$

где $\tilde{\eta}\left(\omega_{q}\right)$ - функция распределения фононов. Подставляя $S_{0}$ в $(22 \mathrm{a})$, в области температур $T \gtrsim \theta_{\mathrm{D}}$ получаем

$$
\tilde{A}_{0}=\frac{1}{4 \pi} \frac{1}{v_{0} m} \bar{\Omega} e^{-2 W} \int d q q^{3}|\widetilde{V}(q)|^{2} \tilde{\eta}\left(\omega_{q}\right)=C_{4}\left|\widetilde{V}\left(q_{\mathrm{D}}\right)\right|^{2} n_{\mathrm{c}}^{1 / 3} \frac{1}{v_{0} m} \frac{T}{\theta_{\mathrm{D}}} e^{-2 W},
$$

где $C_{4}=12$. Выражение (23) написано в модели Дебая с линейным законом дисперсии, так что $q_{\mathrm{D}}$ - дебаевский волновой вектор.

В той же последовательности вычисляются поправки более высокого порядка по потенциалу рассеяния к члену (23). Например, вычислим вклад в корреляционную функцию (20в) порядка $|\widetilde{V}(q)|^{2}$ и соответствуюшую поправку в (20б). Затем подставим полученное выражение в (22a). В результате после ряда преобразований находим

$$
\tilde{A}_{2}=\frac{1}{\pi} \frac{1}{v_{0} m} \bar{\Omega} e^{-2 W} \int d q q^{3}\left[\frac{1}{\omega_{q}^{2}}|\widetilde{V}(q)|^{2}\right]^{2} \frac{1}{\omega_{q}} \int_{0}^{\Delta_{q}} d \widetilde{\omega} \widetilde{\omega}\left(\Delta_{q}-\widetilde{\omega}\right) \eta(\widetilde{\omega}) \eta\left(\Delta_{q}-\widetilde{\omega}\right),
$$

где $\Delta_{q}$ - энергия, переданная частице при поглошении (испускании) двух фононов.

Поскольку энергетические потери быстрых частиц (19) пропорциональны $\left|V_{\mathrm{c}}\right|^{2}$, их следует сравнивать с (23). Учитывая, что $T>\theta_{\mathrm{D}}$, воспользуемся высокотемпературной аппроксимацией фактора Дебая-Валлера в (23), тогда потери становятся функцией температуры вида

$$
\tilde{A}_{0} \sim \frac{T}{\theta_{\mathrm{D}}} \exp \left(-C_{5} \frac{T}{\theta_{\mathrm{D}}}\right) .
$$

4 Теоретическая и математическая физика, т. 140, № 1, 2004 г. 
Тепловые колебания вызывают флуктуации плотности атомов решетки. В результате вероятность рассеяния фононов становится пропорциональной квадрату амплитуды флуктуаций, а следовательно, температуре. Линейный по температуре множитель в (24) ослабляет экспоненциальный спад величины $\tilde{A}_{0}(24)$, который дает фактор Дебая-Валлера, описывающий вклад многофононных процессов в динамическое трение. Таким образом, формула динамической теории дает лишь слабый спад потерь с ростом температуры. Динамическая теория (23), (24), с одной стороны, качественно подтверждает температурную зависимость (19), полученную на базе модели с "замороженным" разбросом атомов, с другой - она раскрьвает механизм ослабления температурного хода кинетического коэффициента.

\section{5. ТРИ РЕЖИМА ДВИЖЕНИЯ ЧАСТИЦ: КАНАЛИРОВАНИЕ, КВАЗИКАНАЛИРОВАНИЕ И ХАОТИЧЕСКОЕ ДВИЖЕНИЕ}

Согласно нелокальной теории [5], [15] при углах влета в канал $\psi_{0}<\psi_{\text {cr пучок частиц }}$ разбивается на две части: каналированную и хаотическую. Каналированные частицы движутся в центре канала вдоль кристаллографической плоскости, образующей стенку канала. Хаотические частицы движутся по прямой траектории под произвольным углом к стенке.

Более сложную картину дает локальная теория, построенная на основе локальной матрицы случайных воздействий $(2),(6)$. Результаты этой теории свидетельствуют о наличии трех режимов движения (рис. 2), поскольку она разделяет направленный пучок на две фракции: хорошо каналированные частищы с малыми поперечными энергиями $\varepsilon_{\perp}<\chi_{i}$ и квазиканалированные частицы с энергиями $\varepsilon_{\perp} \sim \chi_{i}$. Зона квазиканалирования энергетически примыкает к вершине потенциального барьера плоскости [16]. Поэтому в режиме квазиканалирования частицы движутся по фокусированным траекториям [17] вдоль кристаллографической плоскости, но в отличие от каналированных частиц - в непосредственной близости от стенки канала.

Особенности кинетических функций $a\left(\varepsilon_{\perp}\right)$ и $b\left(\varepsilon_{\perp}\right)$, показанные на рис. 2 , можно связать с дополнительным вкладом от квазиканалирования. Безразмерные энергетические потери $a\left(\varepsilon_{\perp}\right)$ имеют максимумы в точках $\chi_{i}$ (с изломом функции в точке максимума). В тех же точках безразмерная диффузионная функция имеет излом, причем на рис. 2 видны конечные разрывы производных в точке $\chi_{i}$. В отличие от результата представленной нами локальной теории нелокальная теория [15] дает гладкие монотонно возрастающие кинетические функции, не имеющие никаких особенностей.

Наличие пика функции энергетических потерь $a\left(\varepsilon_{\perp}\right)$ является самым заметным результатом локальной теории. Этот пик имеет естественное объяснение, поскольку квазиканалированнные частицы движутся вблизи стенки канала, где динамическое трение, обусловленное тепловыми смешениями атомов кристаллографической плоскости, является максимальным. Что же касается уменьшения значения $\chi(T)$ с ростом температуры, то оно обусловлено уменьшением величины плоскостного потенциала и, как следствие, сдвигом энергетической полосы в сторону меньших значений поперечной энергии.

Наконец, сделаем одно замечание, касающееся потерь хаотического пучка. В области $\varepsilon_{\perp}>\varepsilon_{\perp}^{\mathrm{cr}}$ функция $a\left(\varepsilon_{\perp}\right)$ с ростом поперечной энергии асимптотически приближается 
к прямой $C D$ на рис. 2 , которая соответствует тормозной способности аморфной среды $a_{\text {amorf. }}$ Если принять во внимание максимумы энергетических потерь при $\varepsilon_{\perp}=\chi_{i}$, то можно считать, что локальная теория показала наличие более сильного торможения, чем торможение аморфной среды.

В заключение отметим, что теоретические результаты, касающиеся энергетических потерь (в том числе наличие максимума за счет квазиканалирования) согласуются с данными экспериментальных наблюдений [18], [19].

\section{Список литературы}

[1] J. G. Kirkwood. J. Chem. Phys. 1946. V. 14. P. 180

[2] J. G. Kirkwood. J. Chem. Phys. 1946. V. 15. P. 72.

[3] A. Suddaby. P. Gray. Proc. Phys. Soc. A. 1960. V. 75. P. 109.

[4] Д. Н. Зубарев. Неравновесная статистическая термодинамика. М.: Наука, 1971.

[5] J. Lindhard. Mat. Fyz. Medd. Dan. Selek. 1965. V. 34. № 14. P. 1.

[6] M. Rahman. Phys. Rev. B. 1996. V. 52. № 5. P. 3383.

[7] M. Medina Noyola, J. Keizer. Physica A. 1981. V. 107. P. 437.

[8] Джс. Кайзер. Статистическая термодинамика неравновесных процессов. М.: Мир, 1990.

[9] В. И. Тихонов, М. А. Миронов. Марковские процессы. М.: Советское радио, 1977.

[10] P. Кубо. Некоторые вопросы статистическо-механической теории необратимых процессов. В сб.: Термодинамика необратимых процессов. Ред. Д. Н. Зубарев. М.: ИЛ, 1962. С. 345.

[11] F. J. Wegner. Phys. Rev. B. 1972. V. 5. P. 4529.

[12] Р. Балеску. Равновесная и неравновесная статистическая механика. Т. 2. Гл. 21. М.: Мир, 1978.

[13] Yu. A. Kashlev, N. M. Sadykov. Phys. Stat. Sol. B. 1996. V. 196. P. 19.

[14] Yu. A. Kashlev. Phys. Stat. Sol. B. 1995. V. 190. P. 379.

[15] T. Oshiyama. J. Phys. Soc. Japan. 1980. V. 49. № 1. P. 290.

[16] L. T. Chadderton. Rad. Eff. 1975. V. 27. № 1. P. 12.

[17] P. Jonk, B. Schmeiedeskamp, H. E. Roosendaal, H. O. Lutz. Nucl. Instr. Methods B. 1989. V. 43. P. 9.

[18] H.E. Roosedaal, W. H. Kool, W.F. Van Der Weg, J. B. Sanders. Rad. Eff. 1974. V. 22. P. 89.

[19] S. Kopta, R. Hajduk, J. Lekki, B. Rajchel, A. Hrynkiewicz. Phys. Stat. Sol. A. 1989. V. 113. P. 295.

Поступила в редакцию 03.III.2003 г., после доработки 10.VI.2003 г. 